\section{Desarrollo de un nuevo tratamiento para la conservación de piedras con alto contenido en carbonatos utilizando la Tecnología de Resinas de Intercambio lónico}

\author{
M. Pérez-Alonso* , K. CAStro* ${ }^{*}$ M. a D. Rodriguez-Laso** , J.M. Madariaga* \\ * Kimika Analitikoaren Saila, ${ }^{* *}$ Pintura Saila \\ Euskal Herriko Unibertsitatea (UPV/EHU)
}

\begin{abstract}
Resumen
La contaminación atmosférica es la principal causa de deterioro de la piedra monumental presente en el medio urbano. La emisión de gases produce una acidificación gradual del medio reduciendo la resistencia de todos los tipos de piedra. Las piedras con alto contenido en $\mathrm{CaCO}_{3}$ (calizas, mármoles y areniscas carbonatadas) son las más afectadas por la lluvia ácida. Numerosos estudios llevados a cabo sobre la influencia del oxalato de calcio en piedras calcáreas concluyen que este compuesto, producido de forma natural por algunos líquenes, puede actuar como protección frente al ataque ácido. El oxalato de calcio es más estable que el carbonato de calcio frente a los cambios de $\mathrm{pH}$. El tratamiento propuesto en este trabajo consiste en la conversión inducida de forma artificial del carbonato de calcio presente en piedras calcáreas en oxalato cálcico utilizando para ello la tecnología de intercambio iónico. La resina puesta en su forma oxalato debe ser puesta en contacto con la piedra mediante una solución conductora de iones, produciendo la formación de una microcapa cristalina de oxalato cálcico sobre la superficie de la piedra. Los resultados se han evaluado mediante espectroscopia FT-Raman. Los cristales formados inicialmente son $\mathrm{CaC}_{2} \mathrm{O}_{4} \cdot 2 \mathrm{H}_{2} \mathrm{O}$ (weddellita) que evoluciona parcialmente hacia la especie $\mathrm{CaC}_{2} \mathrm{O}_{4} \cdot \mathrm{H}_{2} \mathrm{O}$ (whewellita) en función de las condiciones atmosféricas (humedad y temperatura).
\end{abstract}

Palabras clave: Conservación, piedra, calcárea, intercambio iónico, oxalato.

\begin{abstract}
Atmospheric contamination is the main cause of erosion of stone in urban monuments. Gas emissions produce a gradual acidification in the environment, reducing the resistance of all types of stone. Stones with high $\mathrm{CaCO}_{3}$ content (limestone, carbonated marble and sandstone) are those most affected by acid rain. Numerous studies into the influence of calcium oxalate on calcareous stones conclude that this compound, produced naturally from certain lichens, can act as protection against acid. Calcium oxalate is more stable than calcium carbonate against changes in $\mathrm{pH}$. The treatment proposed in this study consists of the artificially induced conversion of calcium carbonate present in calcareous stones into calcium oxalate using ionic exchange technology. Resin in oxalate form is placed in contact with the stone through an ion conducting solution, producing the formation of a crystalline micro-layer of calcium oxalate over the surface of the stone. The results have been evaluated with FT-Raman spectroscope. The crystals formed initially are $\mathrm{CaC}_{2} \mathrm{O}_{4} \cdot 2 \mathrm{H}_{2} \mathrm{O}$ (weddellite) which evolves partially into the species $\mathrm{CaC}_{2} \mathrm{O}_{4} \cdot \mathrm{H}_{2} \mathrm{O}$ (whewellite) according to atmospheric conditions of humidity and temperature.
\end{abstract}

Key words: Conservation, stone, calcareous, ionic exchange, oxalate.

\section{INTRODUCCIÓN}

El término meteorización de la piedra («stone weathering») se refiere a una combinación de interacciones químicas, físicas y biológicas que ocurren entre la piedra y la atmósfera. Este fenómeno es observable tanto en yacimientos naturales así como en la piedra utilizada como material de construcción (Del MonTe, SABBioni, 1986). El proceso de deterioro puede tener lugar a través de cuatro mecanismos diferentes: cristalización de sales conocidas como eflorescencias (NORD, 1992), ataque de gases ácidos presentes en la atmósfera, ciclos de frío-calor y acción de microorganismos (CHEN et alii, 2000).

La contaminación atmosférica es actualmente la principal causa de deterioro de la piedra monumental presente en el medio urbano. La acidificación gradual del medio ambiente causada por la emisión de gases (particularmente $\mathrm{SO}_{2}$ y $\mathrm{NO}_{\mathrm{x}}$ ) reduce la resistencia de todos los tipos de piedra incluidos los que aparentemente han permanecido intactos durante años. Cuando llueve los gases atmosféricos se disuelven en agua y son parcialmente oxidados formándose ácido sulfúrico y nítrico, así como los ácidos no oxidados sulfuroso y nitroso. Estos ácidos atacan al carbonato de la piedra produciendo las correspondientes sales cálcicas de sulfato, sulfito, nitrato y nitrito (ZAPPIA et alii, 1998).

El agua de lluvia debe entenderse como un elemento activo en el mecanismo químico y físico del deterioro. Proporciona el medio necesario para que se produzcan reacciones químicas tanto en la superficie como en el interior. La cristalización de sales como $\mathrm{NaCl}$ (origen natural) y yeso (por sulfatación del carbonato cálcico) dentro de los poros de la piedra ha sido ampliamente citada como causa de desintegración (Del MonTe, 1992). Cuando la calcita $\left(\mathrm{CaCO}_{3}\right)$ se transforma químicamente en yeso $\left(\mathrm{CaSO}_{4} \cdot 2 \mathrm{H}_{2} \mathrm{O}\right)$, el volumen molar incrementa en un factor de 2 , causando tensión interna, roturas y exfoliación. El yeso es particularmente soluble en el agua de lluvia, pero la calcita también puede disolverse de acuerdo con la reacción química (NORD, Tronner, 1995).

$$
\begin{array}{r}
\mathrm{CaCO}_{3}(\mathrm{~s})+\mathrm{H}_{2} \mathrm{O}(1)+\mathrm{CO}_{2}(\mathrm{~g}, \mathrm{aq}) \\
\mathrm{Ca}^{2+}(\mathrm{aq})+2 \mathrm{HCO}_{3}^{-}(\mathrm{aq})
\end{array}
$$

En su fase de crecimiento los cristales pueden atrapar partículas en suspensión de minerales, carbón y hollín dando lugar a la formación de incrustaciones negras.

Un gran número de investigaciones han tenido lugar en Italia en relación con el origen de las incrustaciones negras (CAMUfFo et alii, 1982), (FASSINA, 1991), (AUSSET et alii, 1992). Estos estudios han demostrado que junto con las partículas de carbón y hollín, (procedentes de la com- 
bustión de combustibles fósiles) las incrustaciones contienen oxalato cálcico $\left(\mathrm{CaC}_{2} \mathrm{O}_{4}\right.$ en diferentes estados de hidratación), cristales de yeso y óxidos de metales que catalizan la oxidación de $\mathrm{SO}_{2}$ y promueven el crecimiento de las incrustaciones. Trabajos recientes han demostrado que el sulfato existente en las incrustaciones negras proporciona la fuente nutricional necesaria para el crecimiento de algunos microorganismos (ORTEGA et alii, 1994) lo que implica que dichas incrustaciones negras constituyen emplazamientos adecuados para la proliferación de microorganismos.

Las piedras calizas y el mármol (formado mayoritariamente por $\mathrm{CaCO}_{3}$ ), así como las areniscas con alto contenido en carbonatos son las más vulnerables al ataque ácido y por lo tanto las más propensas a fijar partículas atmosféricas que den lugar a la formación de zonas ennegrecidas. Debido al gran número de piedras calizas y sedimentarias calcáreas, incluso mármol en algunos casos, presentes en las fachadas de edificios de interés histórico es necesario el desarrollo de un tratamiento para su conservación.

En la bibliografía se ha discutido con profundidad el origen y la influencia del oxalato cálcico en la superficie de las piedras usadas en la construcción de edificios. Algunos autores (GUIDOBALDI et alii, 1985), (FrANZINI et alii, 1984), afirman que el ácido oxálico es el producto de descomposición de materia orgánica usada como tratamiento superficial por motivos estéticos o protectores. Por el contrario trabajos más recientes (DEL MONTE et alii, 1987), (EDWARDS, 1991) presentan la hipótesis de que la precipitación del oxalato cálcico se debe a la acción del ácido oxálico secretado por microorganismos tales como líquenes o algas azules que colonizan los monumentos. El ácido oxálico por ataque al carbonato de calcio precipita como oxalato de calcio. En dichos estudios se concluye que el oxalato cálcico producido por algunos líquenes de forma natural puede actuar como medio de protección de las piedras frente al ataque ácido. En las microcapas cristalinas que se forman naturalmente la fina membrana formada durante siglos es muy compacta y puede obstruir la libre difusión de sales solubles, aunque el oxalato de calcio en sí es hidrofílico (MATTEINI et alii, 1994).

La conversión de $\mathrm{CaCO}_{3}$ en $\mathrm{CaC}_{2} \mathrm{O}_{4}$ ha dado lugar en Italia a diversos experimentos. Matteini y sus colaboradores se enfrentaron al problema de proteger las pinturas murales de los contaminantes atmosféricos y para ello utilizaron oxalato amónico (MATTEINI, 1987). El estudio de las pátinas de oxalato formadas concluyó cómo éstas protegen la superficie formando un caparazón fino, compacto y no poroso. Esto ocurre como consecuencia de la mayor estabilidad química del oxalato de calcio sobre el carbonato de calcio frente a los cambios de $\mathrm{pH}$. $\mathrm{El} \mathrm{CaCO}_{3}$ se ve atacado a $\mathrm{pH}$ inferiores a 6, mientras que el $\mathrm{CaC}_{2} \mathrm{O}_{4}$ soporta hasta $\mathrm{pH}$ ligeramente inferiores a 2 . A continuación se muestran las reacciones implicadas en el proceso propuesto para la conservación de las pinturas murales:

$$
\begin{gathered}
\mathrm{CaCO}_{3}+\left(\mathrm{NH}_{4}\right)_{2} \mathrm{C}_{2} \mathrm{O}_{4} \\
\mathrm{CaC}_{2} \mathrm{O}_{4} \cdot \mathrm{H}_{2} \mathrm{O}+\mathrm{HCO}_{3}^{-}+\mathrm{NH}_{3}+\mathrm{NH}_{4}^{+}
\end{gathered}
$$

La capa mineral formada sobre la superficie es químicamente compatible con el carbonato de calcio que constituye la piedra y difícilmente meteorizable, evolucionando en función de las condiciones climáticas entre dos estructuras químicas diferentes (whewellita $\mathrm{CaC}_{2} \mathrm{O}_{4} \cdot \mathrm{H}_{2} \mathrm{O}$ y weddellita $\left.\mathrm{CaC}_{2} \mathrm{O}_{4} \cdot 2 \mathrm{H}_{2} \mathrm{O}\right)$.

Asimismo la comparación de las solubilidades de oxalato cálcico y yeso sugiere que el oxalato amónico sería también capaz de transformar el yeso en whewellita $\left(\mathrm{CaC}_{2} \mathrm{O}_{4} \cdot \mathrm{H}_{2} \mathrm{O}\right)$ gracias a un mecanismo de doble intercambio como el considerado anteriormente.

$$
\begin{aligned}
& \mathrm{CaSO}_{4} \cdot 2 \mathrm{H}_{2} \mathrm{O}+\left(\mathrm{NH}_{4}\right)_{2} \mathrm{C}_{2} \mathrm{O}_{4} \\
& \mathrm{CaC}_{2} \mathrm{O}_{4} \cdot \mathrm{H}_{2} \mathrm{O}+\left(\mathrm{NH}_{4}\right)_{2} \mathrm{SO}_{4}+\mathrm{H}_{2} \mathrm{O}
\end{aligned}
$$

El tratamiento propuesto en este trabajo consiste en la inducción artificial de la transformación de la superficie de carbonato cálcico en oxalato cálcico mediante la aplicación de resinas de intercambio iónico. El uso de resinas de intercambio iónico tiene como fin evitar la presencia de un exceso de oxalato en la disolución, que podría generar complejos coloreados si la piedra en estudio tuviera gran porcentaje de hierro y otros metales de transición tal y como ocurre en los experimentos llevados a cabo en Inglaterra. Cezar basándose en los trabajos previos de Matteini utilizó oxalato amónico para tratar diferentes muestras de piedra caliza. En este caso el oxalato amónico actúa como quelante. El hierro soluble y el carbonato de calcio reaccionan con el oxalato amónico, formándose dos productos de forma simultánea, oxalato de calcio y oxalato de hierro. Éste último provoca cambios en la coloración de la piedra (CEZAR, 1998).

La resina fuertemente básica en su forma oxalato debe ser puesta en contacto con la piedra por medio de una disolución conductora de iones. La optimización de esta disolución (componentes y concentraciones), es el paso más crítico del proceso siendo de vital importancia la elección de su $\mathrm{pH}$ y naturaleza química. Si el contacto entre la resina y la superficie de la piedra es el adecuado comienza el intercambio iónico, donde los iones oxalato reaccionan con los cationes calcio para formar una pátina de oxalato cálcico sobre la superficie. A continuación se muestra un esquema del mecanismo que tiene lugar en el proceso. 


\begin{tabular}{llll}
\hline & \multicolumn{1}{c}{ Superficie } & Disolución & \multicolumn{1}{c}{ Resina } \\
\hline Situación inicial: & $\mathrm{CaCO}_{3}$ piedra & $\mathrm{C}_{2} \mathrm{O}_{4}^{2-}$ & $\mathrm{C}_{2} \mathrm{O}_{4}-\mathrm{R}_{2}$ \\
Mecanismo: & $\mathrm{CaCO}_{3}$ piedra $+\mathrm{CaC}_{2} \mathrm{O}_{4}$ & $\mathrm{CO}_{3}^{2-}$ & $\mathrm{C}_{2} \mathrm{O}_{4}-\mathrm{R}_{2}$ \\
Situación final: & $\mathrm{CaCO}_{3}$ piedra $+\mathrm{CaC}_{2} \mathrm{O}_{4}$ & $\mathrm{C}_{2} \mathrm{O}_{4}^{2-}$ & $\mathrm{C}_{2} \mathrm{O}_{4}-\mathrm{R}_{2}+\mathrm{CO}_{3}-\mathrm{R}_{2}$ \\
\hline
\end{tabular}

Teóricamente cuando un carbonato sale de la piedra entra a su superficie un oxalato; si ese carbonato es intercambiado en la resina por otro oxalato, el balance neto es la presencia de la misma masa de oxalatos en la disolución de contacto piedra/resina actuando la resina como reservorio de oxalatos que los va dosificando sólo cuando son captados los carbonatos que salen de la piedra.

\section{DESARROLLO EXPERIMENTAL}

Los resultados se evaluaron mediante espectroscopia FT-Raman usando un espectrómetro Nicolet FT-Raman 950, que incorpora un láser de $1064 \mathrm{~nm}$ (Nd:YAG) y un detector InGaAs. La adquisición de datos se llevó a cabo con el software Omnic de Nicolet. Con ayuda del portamuestras para superficies planas se registraron espectros FT-Raman sobre un spot de $0.5 \mathrm{~mm}$ de diámetro.

Para la realización del estudio se prepararon cinco probetas de piedra caliza recogidas en el medio natural en una zona de escasa contaminación atmosférica (Pozalagua, Carranza). En la figura 1, se muestra el espectro Raman de una de las probetas utilizadas en el ensayo junto con el espectro de un patrón de carbonato cálcico (bandas características a $\left.1085,711,280,154 \mathrm{~cm}^{-1}\right)$. En el espectro de la probeta aparece la típica señal fluorescente (curvatura que produce la desviación de la línea base) debida a impurezas que dan a la piedra su color grisáceo característico. Sin embargo en dicha probeta es posible identificar los picos ca-

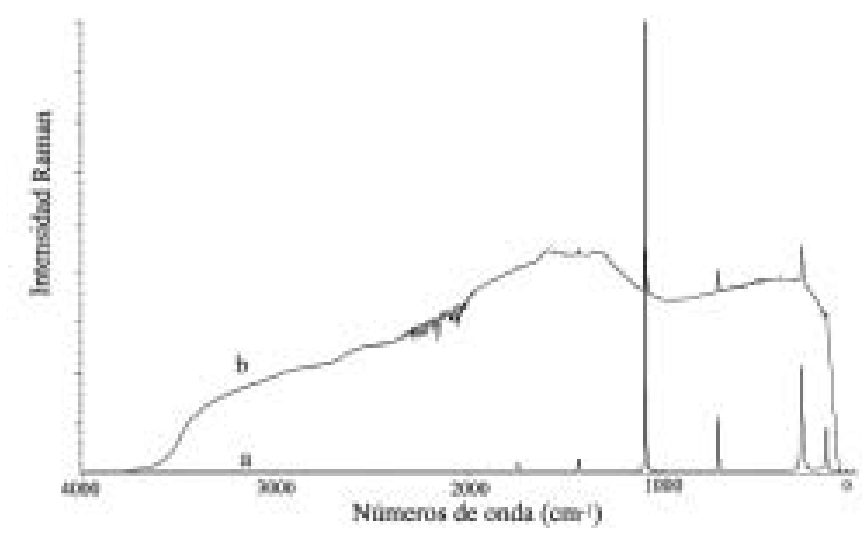

Fig. 1. Estudio de la composición estructural de las probetas empleadas en el estudio mediante espectroscopia FT-Raman. a) Espectro patrón de carbonato cálcico $\left(\mathrm{CaCO}_{3}\right)$; b) Espectro de una probeta de piedra caliza antes del tratamiento racterísticos de carbonato de calcio pudiendo concluirse que la piedra es caliza.

Para la realización del estudio se eligió la resina Dowex 550A, una resina de intercambio aniónico fuertemente básica de tipo monoesfera.

Sobre cada probeta se depositaron aproximadamente 10 gramos de la resina acondicionada en su forma oxalato y en contacto con la disolución conductora elegida. Periódicamente se fueron añadiendo gotas de la disolución de contacto con el fin de evitar la cristalización de oxalato superficial por evaporación.

Se establecieron 4 tiempos distintos de contacto entre la resina y las probetas: 6 horas, 24 horas, 48 horas y 72 horas.

Cada probeta se retiró al tiempo prefijado. Primeramente se eliminó la resina depositada en la superficie, a continuación se lavaron con agua de calidad MilliQ y se dejaron secar a temperatura ambiente.

\section{RESULTADOS Y DISCUSIÓN}

Para determinar si se había producido algún tipo de reacción se comparó el espectro de la piedra antes del inicio de la reacción, con los espectros obtenidos tras la eliminación de la resina de intercambio aniónico. En la figura 2 puede observarse cómo aparecen nuevas señales que no existían en la piedra antes del contacto en menos de 24 horas de tratamiento.

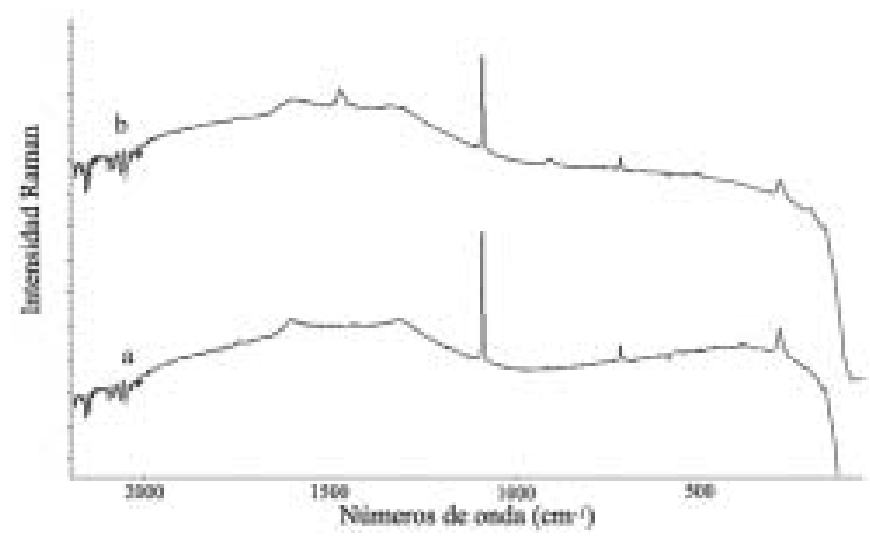

Fig. 2 Aparición de oxalato cálcico dihidratado: a) Espectro Raman de una probeta antes del tratamiento; b) Espectro Raman de una probeta analizada 6 días después de haber sido sometida a un tratamiento durante 48 horas 


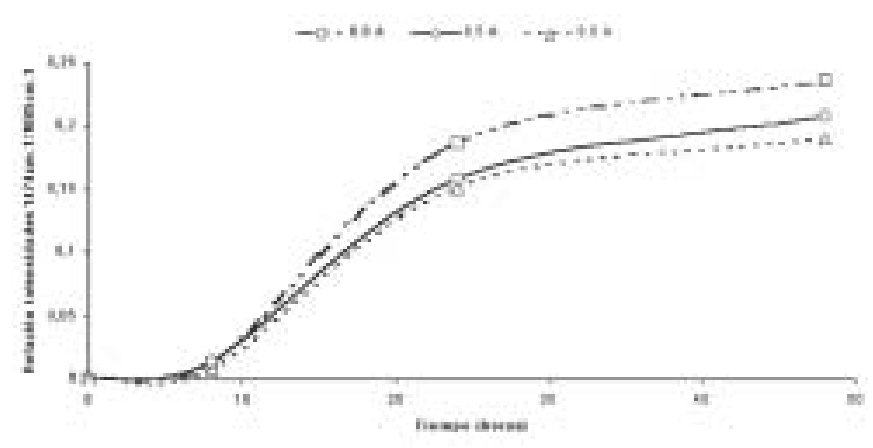

Fig. 3. Relación de señales oxalato cálcico dihidratado $\left(1474 \mathrm{~cm}^{-1}\right) /$ carbonato cálcico $\left(1085 \mathrm{~cm}^{-1}\right)$ en función del tiempo de tratamiento, para tres niveles de potencia de láser

A partir de datos bibliográficos se determinó que la pátina estaba constituida por $\mathrm{CaC}_{2} \mathrm{O}_{4} \cdot 2 \mathrm{H}_{2} \mathrm{O}$ (weddellita, bandas características a $\left.1474,908,506 \mathrm{~cm}^{-1}\right)(\mathrm{E}<$ DWARDS et alii, 1995).

Realizando diversos espectros en distintos puntos de las probetas en estudio y mediante el uso de técnicas estadísticas es posible obtener una idea de la uniformidad de la pátina de oxalato formada.

En la Figura 3 se muestra la variación de la relación de señales a $1474 \mathrm{~cm}^{-1}$ (oxalato de calcio dihidratado)/ 1085 $\mathrm{cm}^{-1}$ (carbonato de calcio) en función del tiempo de aplicación del tratamiento para diferentes intensidades de láser. En la técnica de análisis empleada, la espectroscopia FT-Raman, a medida que aumenta la potencia del láser aumenta también su profundidad de penetración. En la figura 3 se observa cómo al aumentar la potencia del láser la señal del oxalato disminuye con respecto a la de carbonato, claro indicativo de que la reacción se está produciendo únicamente de forma superficial. También puede concluirse cómo para tiempos de tratamiento más largos, la capa de oxalato formada aumenta de espesor. Sin embargo la relación de señales tiende a un valor máximo y constante, debido a un efecto de saturación (en el primer día se forma aproximadamente un $70 \%$ del espesor final alcanzado). El espesor de la capa formada no puede aumentar de forma proporcional al tiempo de aplicación, sino que está restringido por el número de carbonatos accesibles en la superficie de la piedra. Una vez que se ha cubierto completamente la superficie de la piedra el proceso se para sin necesidad de intervención externa.

Los análisis realizados mediante espectroscopia FTRaman se repitieron en las probetas en estudio para comprobar la evolución temporal de la microcapa de oxalato cálcico formada, realizando medidas a varios días de distancia tras haber eliminado la resina de las probetas.

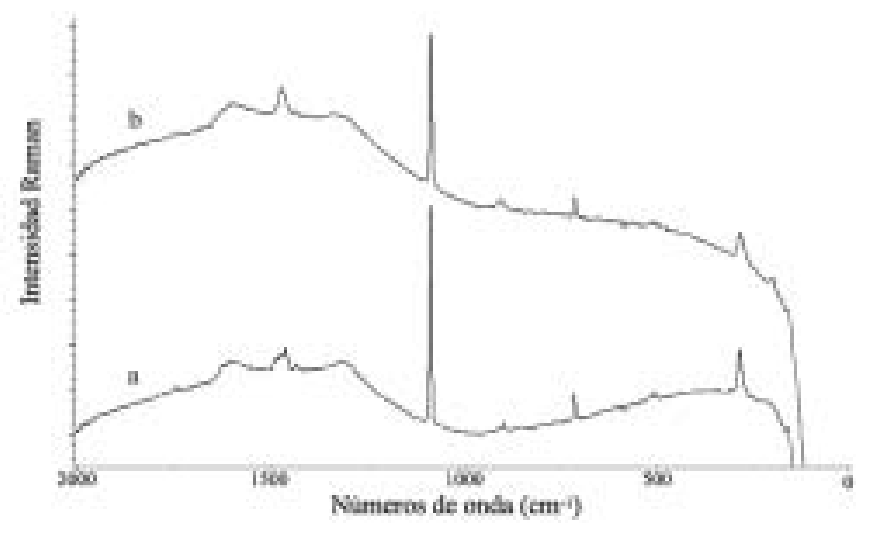

Fig. 4. Evolución cinética de la pátina de oxalato formada en la probeta sometida a tratamiento de 48 horas. a) Espectro Raman 43 días después del tratamiento; b) Espectro Raman 6 días después del tratamiento

La pátina generada de $\mathrm{CaC}_{2} \mathrm{O}_{4} \cdot 2 \mathrm{H}_{2} \mathrm{O}$ (weddellita) no es estable (desde el punto de vista estructural) y dependiendo de las condiciones atmosféricas (humedad y temperatura) se transforma parcialmente en la especie $\mathrm{CaC}_{2} \mathrm{O}_{4} \cdot \mathrm{H}_{2} \mathrm{O}$ (whewellita, claro doblete a 1465 y $1492 \mathrm{~cm}^{-1}$ ) (DuvaL, 1985) (Ver figura 4) sin que por ello se vea afectada su capacidad protectora sobre el carbonato de calcio. La conversión no es total y la proporción relativa de los dos diferentes oxalatos de calcio cambia con el tiempo debido a que las condiciones climatológicas juegan un papel muy importante en la conversión de weddellita en whewellita y viceversa (HOLDER et alii, 2000).

La comparación de las dos estructuras cristalinas para weddellita y whewellita revela grandes similitudes (TAZZOLI, Domeneghetti, 1980) lo que está de acuerdo con la frecuente asociación de los dos minerales y la sencilla transformación de uno en el otro. Como dato significativo de los dos compuestos se presenta la relación en los parámetros de celda $\mathrm{c}_{\text {weddellita }} / \mathrm{b}_{\text {whewellita }}=1 / 2$. La distorsión de la estructura resultado de la deshidratación de la weddellita a whewellita se observa a lo largo de la repetición [201] $]_{\text {whewellita }}$ y $[100]_{\text {weddellita }}$, produciéndose un cambio del sistema de enlace de hidrógeno.

En la figura 5 se observa la evolución temporal de las distintas especies de oxalato cálcico, relativizadas respecto del carbonato de calcio, 43 días después de haberse realizado el tratamiento con la resina. En esta experiencia se utilizaron las probetas que habían sido tratadas durante 6, 24 y 48 horas. Se aprecia claramente como al dejar evolucionar las probetas libremente en la atmósfera del laboratorio y a diferencia de los primeros días cuando únicamente aparecía oxalato de calcio dihidratado, la pátina evoluciona hacia una mezcla de ambos tipos de oxalatos cálcicos. 


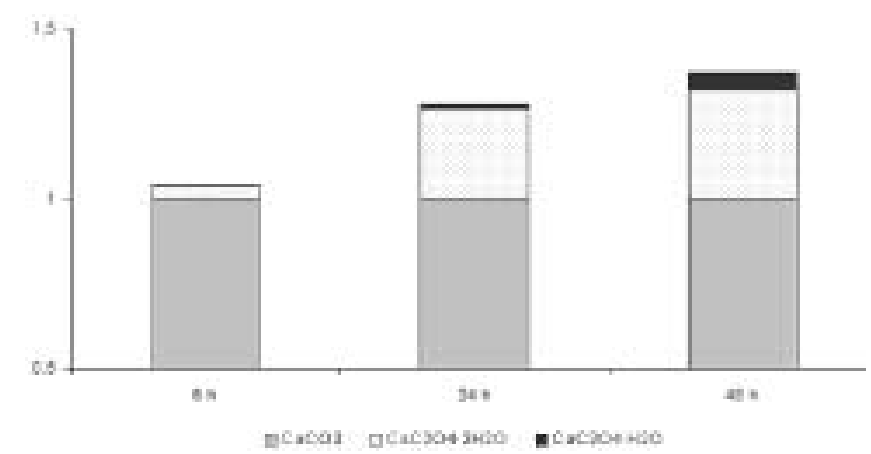

Fig. 5. Estado de las distintas especies de oxalato cálcico, para las probetas sometidas a 6, 24 y 48 horas de tratamiento; a los 43 días de haberse finalizado el tratamiento. Las señales se encuentran relativizadas respecto del carbonato de calcio

\section{CONCLUSIONES}

Con la realización del presente trabajo se ha demostrado la aplicabilidad de la tecnología de intercambio iónico para realizar una mineralización en superficie de las piedras monumentales con alto contenido en carbonatos. La pátina de oxalato cálcico formada actuaría como método de protección frente al ataque de los gases ácidos presentes en todo tipo de atmósfera, respondiendo a su vez de forma activa ante otros cambios atmosféricos de temperatura y humedad.

Sin embargo una vez entendido el proceso de formación de la pátina requerida y sus posibles transformaciones es necesario realizar diversos estudios con mayor profundidad. Para ello debería realizarse el tratamiento en piedras de distintos tipos y características, estudiando las posibles interferencias de otros metales y componentes presentes en las muestras a tratar. Así mismo también deberían realizarse estudios de la estabilidad de la pátina formada frente a cambios de humedad, temperatura y $\mathrm{pH}$, mediante el control de la evolución cinética de la pátina formada bajo condiciones ambientales controladas.

Agradecimientos. M. Pérez-Alonso agradece al Gobierno Vasco su beca Pre-Doctoral. K. Castro agradece al MECD su beca Pre-Doctoral. Este trabajo ha sido financiado a través del proyecto UE02-A06

\section{Bibliografía}

Ausset P., Lefevre R., Phillipon J., Venet C., 1992, Large scale distribution of fly-ash particles inside weathering crusts on calcium carbonate substrates: Some examples on French monuments, La conservazione del monumenti nel bacino del Mediterraneo, ed. D. Decrouez, J. Chamay, and F. Zerra, pp. 121-139, Geneva.

Camuffo D., del Monte M., Sabbioni C., Vittori O., 1982, Wetting, deterioration and visual features of stone surfaces in an urban area, Atmospheric Environment, 16, pp. 2253-2259.
Cezar T.M., 1998, Calcium oxalate: A surface treatment for limestone, Journal of Conservation \& Museum Studies, 4, May (http:// palimpsest.stanford.edu/jcms/issue4/cezar.html).

Chen J., Blume H. P.,Beyer L., 2000, Weathering of rocks induced by lichen colonization. A review, Catena, 39, pp. 121-146.

Del Monte M., Sabbioni C., 1986, Chemical and biological weathering of an historical building: Reggio Emilia Cathedral, The Science of the Total Environment, 50, pp. 165-182.

del Monte M., Sabbioni C., Zappia G., 1987, The origin of calcium oxalates on historical buildings, monuments and natural outcrops, The Science of the Total Environment, 67, pp. 17-39.

DEL MonTe M., 1992, Air pollution and decay of building stone, Analusis, 20, pp. M20-M23.

Duval D., Condrate R.A.,1985, Temperature dependence of the Raman spectra of calcium oxalate monohydrate, Phys: Status Solidi B, 132, pp. 83-92.

EDWARDS H.G.M., 1991, Raman spectra of oxalates in lichen encrustations on Renaissance frescoes, Spectrochimica Acta, Part A, 47A, pp. 1531-1539.

EdWARdS H.G.M., RusSel N.C., SeaWARD M.R.A., SOlarKe D., 1995, Lichen biodeterioration under different microclimates an FT-Raman spectroscopic study, Spectrochim. Acta, Part A, 51A, pp. 2091-2100.

FASSINA V., 1991, Atmospheric pollutants responsible for stone decay: Wet and dry surface deposition of air pollutants on stone and the formation of black scabs, Weathering and Air Pollution, pp. 67-68, Bari.

Franzini M., GratziU C., Wicks E., 1984, Patine ad ossalato di calcio su monumenti marmorei, Sco. Ital. Mineral. Petrog, 39, pp. 59-70.

Guidobaldi F., Tabasso M.L., Meucci C., 1985, Monumenti in marmo di epoca imperiale a Roma: indagine sui residui di trattamenti superficiali, Bolletino d'Arte, pp. 121-134.

Holder J.M., Wynn-Williams D.D., Rull-Perez F., Edwards H.G.M., 2000, Raman spectroscopy of pigments and oxalates in situ with epylithic lichens: Acaraspora from the Antarctic and Mediterranean, New Phytol., 145, pp. 271-280.

MatTeini M., 1987, An assessment of Florentine methods of wall painting conservation based on the use of mineral treatments, The Conservation of Wall Paintings, Proceedings of a symposium organized by the Courtauld Institute of Art and the Getty Conservation Institute, London, July 13-16, pp. 137-148.

Matteini M., Moles A., Giovanni S., 1994, Calcium oxalate as a protective mineral system for wall paintings: methodology and analyses, The conservation of monuments in the Mediterranean Basin, Proccedings of the $3^{\text {rd }}$ International Symposium, Venezia, pp. 155-162.

NORD A.G., 1992, Efflorescence salts on wheathered buildings stone in Sweden, Geologiska Föreningenss $i$ Stockholm Förhandlingar, 114, pp. 423-429.

Nord A.G., Tronner K., 1995, Effect of acid rain on sandstone: The Royal Palace and the Riddarholm Church, Stockholm, Water, Air and Solution, 85, pp. 2719-2724.

Ortega J.J., ARIÑo X., Stal L.J., SAiz C., 1994, Cyanobacterial sulfate colonization from black crust of a historical building, Geomicrobiological Journal, 12, pp.15-22.

TAZzoli V., DomeneGHeTti C., 1980, The crystal structures of whewellite and weddellite: re-examination and comparision, American Mineralogist, 65, pp. 327-334.

Zappia G., Sabbioni C., Riontino C., Gobbi G., Favoni O. 1998: Exposure tests of building materials in urban atmosphere, The Science of the Total Environment, 224, pp. 235-244. 